\title{
Crosstalk between skin inflammation and adipose tissue-derived products: pathogenic evidence linking psoriasis to increased adiposity
}

\author{
Andrea Chiricozzi, Annunziata Raimondo, Serena Lembo, Francesca Fausti, \\ Valentina Dini, Antonio Costanzo, Giuseppe Monfrecola, Nicola Balato, Fabio \\ Ayala, Marco Romanelli \& Anna Balato
}

To cite this article: Andrea Chiricozzi, Annunziata Raimondo, Serena Lembo, Francesca Fausti, Valentina Dini, Antonio Costanzo, Giuseppe Monfrecola, Nicola Balato, Fabio Ayala, Marco Romanelli \& Anna Balato (2016): Crosstalk between skin inflammation and adipose tissuederived products: pathogenic evidence linking psoriasis to increased adiposity, Expert Review of Clinical Immunology, DOI: 10.1080/1744666X.2016.1201423

To link to this article: http://dx.doi.org/10.1080/1744666X.2016.1201423

Published online: 20 Jun 2016.

Submit your article to this journal $\sqsubset$

山ll Article views: 7

View related articles

View Crossmark data $\nearrow$ 
Publisher: Taylor \& Francis

Journal: Expert Review of Clinical Immunology

DOI: $10.1080 / 1744666 X .2016 .1201423$

Crosstalk between skin inflammation and adipose tissue-derived products: pathogenic evidence linking psoriasis to increased adiposity

Chiricozzi Andrea ${ }^{1}$, Raimondo Annunziata ${ }^{2}$, Lembo Serena ${ }^{3}$, Fausti Francesca ${ }^{4}$, Dini Valentina ${ }^{1}$, Costanzo Antonio ${ }^{5}$, Monfrecola Giuseppe ${ }^{2}$, Balato Nicola ${ }^{2}$, Ayala Fabio $^{2}$, Romanelli Marco ${ }^{1}$, Balato Anna ${ }^{6}$.

\section{Affiliations:}

${ }^{1}$ Department of Dermatology, University of Pisa, Via Roma 67, Pisa, Italy.

2Department of Clinical Medicine and Surgery, Section of Dermatology, University of Naples Federico II, Naples, Italy.

${ }^{3}$ Department of Medicine and Surgery, University of Salerno, Salerno, Italy

${ }^{4}$ Skin Biology Laboratory, University of Rome Tor Vergata, Rome Italy

${ }^{5}$ Dermatology Unit, Department of Neuroscience, Mental Health and Sensory Organs (NESMOS), Sapienza University of Rome, Italy.

${ }^{6}$ Department of Advanced Biomedical Sciences, University of Naples Federico II, Naples, Italy.

\section{Corresponding author:}

Dr. Anna Balato, MD, PhD

Department of Advanced Biomedical Sciences University of Naples Federico II Via Pansini, 580131 Napoli,

Italy

Email: annabalato@yahoo.it

Ph: +39-0817462457

Fax: +39-0817462442 


\section{Abstract:}

Introduction: Psoriasis is a chronic skin disorder associated with several comorbid conditions. In psoriasis pathogenesis, the role of some cytokines, including TNF- $\alpha$ and IL-17, has been elucidated. Beside their pro-inflammatory activity, they may also affect glucose and lipid metabolism, possibly promoting insulin resistance and obesity. On the other hand, adipose tissue, secreting adipokines such as chemerin, visfatin, leptin, and adiponectin, not only regulates glucose and lipid metabolism, and endothelial cell function regulation, but it may contribute to inflammation.

Areas covered: This review provides an updated "state-of-the-art" about the reciprocal contribution of a small subset of conventional cytokines and adipokines involved in chronic inflammatory pathways, upregulated in both psoriasis and increased adiposity. A systematic search was conducted using the PubMed Medline database for primary articles.

Expert Commentary: Because psoriasis is associated with increased adiposity, it would be important to define the contribution of chronic skin inflammation to the onset of obesity and vice versa. Clarifying the pathogenic mechanism underlying this association, a therapeutic strategy having favorable effects on both psoriasis and increased adiposity could be identified. 
Keywords: psoriasis, obesity, metabolic syndrome, IL-17, TNF- $\alpha$, IL-6, leptin, adiponectin, chemerin, visfatin

\subsection{Introduction}

Psoriasis is a chronic inflammatory skin disease affecting about $2.5 \%$ of the population worldwide [1]. In genetically predisposed subjects, environmental or endogenous stimuli trigger the onset of the disease, characterized by an altered immune activation and an aberrant keratinocyte proliferation [2]. Substantial evidence recognized an association between psoriasis and various comorbid conditions including arthritis, osteoporosis, non alcoholic fatty liver disease, increased spleen longitudinal diameter, cardiovascular diseases, and metabolic syndrome, which constitutes a cluster of disorders ranging from hypertension, central obesity, atherogenic dyslipidemia, and glucose intolerance [3-5]. Patients suffering from psoriasis showed an increased risk of developing metabolic syndrome [6-12]. A higher prevalence of metabolic syndrome was observed in a psoriasis population adjusted for age, sex, race/ethnicity, smoking, and Creactive protein level [13]. The biologic mechanism is not fully understood, though some pathogenic links between these two disorders have been identified. Firstly, some genetic loci represent susceptibility factors (PSORS2-4, CDKAL1, and ApoE4) for both psoriasis and metabolic syndrome [14,15]. Additionally, Western dietary pattern is included among the environmental dietary factors involved in the pathogenesis of both disorders. Differences in dietary intake were observed in adult male psoriatic patients compared to controls. These differences were associated to the severity of psoriasis and cardio-metabolic risk 
$[16,17]$. In obese psoriatic patients, an energy-restricted diet designed to increase n-3 and to reduce n-6 polyunsaturated fatty acids (PUFAs), ameliorated the metabolic profile and, by increasing the response to immuno-modulating therapy, improved the clinical outcomes of the disease [18]. Moreover, metabolic syndrome is characterized by a state of chronic systemic inflammation sustained by the adipose tissue, that is not a mere inert fat storage but an endocrine organ secreting multiple mediators, named "adipokines", contributing to inflammation, as well as to glucose and lipid metabolism, and endothelial cell function regulation [19]. Traditionally, two types of adipose tissue are recognized: brown adipose tissue (BAT), associated with energy expenditure as well as thermogenesis and white adipose tissue (WAT), responsible for energy storage [20]. In addition to adipocytes, adipose tissue also contains endothelial cells, fibroblasts, macrophages, myeloid cells and T cells [21]. In a lean state, $\mathrm{T}$ helper (h)2 cells, T regulatory (reg), Breg, and invariant natural killer (NK)T cells are the dominant cell population in WAT $[22,23]$; in contrast, augmented adiposity results in increased number of pro-inflammatory immune cells, including Th1 and Th17 cells $[22,24]$. Adipokines are able to orchestrate the interaction between metabolic and immune systems. The wide array of adipokines, secreted by the adipose tissue, includes at least 50 bioactive molecules [25], not all exclusively produced by adipocytes. In particular, resident macrophages contribute to the production of some cytokines and chemokines such as interleukin (IL)-1 $\beta$, IL-6, Tumor necrosis factor (TNF)- $\alpha$ and monocyte chemotactic protein(MCP)-1 [26], while more conventional adipokines, such as adiponectin, leptin, visfatin, chemerin, plasminogen activator inhibitor type 1 (PAI-1), are mostly released by adipocytes. Either the mediators released by 
adipocytes or by other cells resident in the adipose tissue, may play an important role in multiple autoimmune skin diseases, acting on immune cells and keratinocytes (Table 1) [27-45] . Notably, TNF- $\alpha$ is highly expressed in both psoriasis and obesity [46], and similarly, other adipokines (i.e., IL-6 and leptin) are known as proinflammatory mediators and may contribute to psoriasis inflammation [3]. Meanwhile, the same pro-inflammatory mediators (i.e., TNF- $\alpha$ and IL-6) may (i) impair glucose metabolism, inducing insulin resistance, or they may lead to (ii) endothelial dysfunction, (iii) altered lipid metabolism, (iv) hypertension, and ( $v$ ) enhanced risk of cardiovascular diseases [46]. However, in a context of chronic adipose tissue inflammation, characterizing obesity, associated or not with insulin resistance, also the adaptive immunity is indeed crucial. In fact, as mentioned above, in augmented adiposity, accumulation of $\mathrm{T}$ cell subsets producing IL-17, IL-22, IL-10 and interferon(IFN)- $\gamma$ is detected [45,47-49]. Particularly, the number of IL-17- or IFN- $\gamma$-producing $\mathrm{T}$ cells positively correlated with adiposity [45,47-49]. These findings reflect some observations from obese mice models showing marked infiltration of both CD4+ and CD8+ T/ cells in adipose tissue, conversely to lean mice, predominantly characterized by a Th2- and Treg-oriented immune activation [50]. Furthermore, diet-induced obese mice and obese human subjects showed elevated IL-6 levels, which predispose to the Th17 lineage expansion, resulting in an increased number of IL-17-secreting CD4+ cells [24]. As mentioned above, psoriasis increases the risk of developing obesity but, concurrently, obesity has been associated with an increased prevalence and severity of psoriasis, even in pediatric population $[6,51,52]$. The fact that obesity may aggravate psoriasis severity has been also confirmed by obese mice model developing exacerbated 
psoriasis-like lesions after imiquimod induction [53]. In this model, obesity worsened skin lesions enhancing the expression of key-cytokines and their downstream gene products, particularly IL-17A, IL-22, IL-23p19, IL-17C, and $\beta$ defensin 3 [53]. Additionally, obesity may negatively affect clinical response to systemic antipsoriatic treatment, and, conversely, weight loss improves psoriasis as well as the therapeutic response [54-56].

Albeit some evidence suggested the potential role of pro-inflammatory psoriasissignature cytokines in obesity pathogenesis, and, similarly, the role of adipokines in psoriasis pathogenesis, the mechanisms linking psoriasis and obesity, and more in general metabolic syndrome, are not fully investigated. For instance, the association between psoriasis and adipokines is reported, though evidence associating psoriasis or disease severity with altered circulating adipokine levels is controversial. Takahashi et al detected a significant negative correlation between plasma adiponectin levels and PASI score, whereas plasma leptin levels were enhanced with increasing PASI score, though not statistically significant [57]. Along these lines, TNF- $\alpha$ plasma concentration negatively and significantly correlated with plasma adiponectin levels, whereas plasma leptin levels were not correlated [57]. Conversely, in another study, both serum adiponectin and leptin levels were significantly decreased in psoriatic patients in comparison with the control group [58]. Other reports detected significantly lower levels of adiponectin and visfatin, and significantly higher levels of vaspin, omentin, chemerin, and resistin in psoriatic patients as compared to the control group [42,59-62]. Of note, a meta-analysis including nine case-control studies, containing 421 psoriasis patients and 348 healthy controls, revealed a statistically significant association between serum resistin levels and psoriasis 
[59]. Interestingly, successful treatment of psoriasis with biologicals (i.e., infliximab and ustekinumab) or phototherapy (narrow band-UVB) was related to an increase of adiponectin serum levels and a decrease of leptin and resistin serum concentration [60]. Coban et al. observed higher levels of chemerin in patients than in controls and a strongly positive correlation between the PASI score and chemerin [61]. Moreover, psoriatic patients showing an improvement in the PASI score correlating with a significant decrease in serum leptin, omentin, and chemerin values, and a significant increase of serum adiponectin levels [61]. Conversely, Gisondi et al. found a significant decrease in the PASI score and serum chemerin levels, but no correlation was identified between these two parameters [42].

Overall, a general agreement regarding the circulating adipokine levels and their pathogenic relevance in psoriasis patients needs to be defined.

Thus, this review is aimed to provide an updated "state-of-the-art" about the reciprocal contribution of a small subset of conventional cytokines and adipokines involved in chronic inflammatory pathways, upregulated in both psoriasis and increased adiposity. The main effects of psoriasis-signature cytokines on increased adiposity as well as of adipokines on psoriasis pathogenesis have been summarized in Table 2. According to the current pathogenic model, we selected the most pathogenically relevant cytokines. IL-17 is recognized as the key cytokine in psoriasis [63]. The main sources of IL-17 involved in psoriasis pathogenesis, namely Th17 cells, Tc17 cells, $\gamma / \delta$ T cells, mast cells, and innate lymphoid cells (ILC)-3 also express IL-22 that synergistically potentiates IL-17 effects [64]. IL-23, the most potent inducer of IL-17/IL-22 expression, was excluded as it acts on immune cells but it does not 
on tissue cells. Indeed, the development of psoriasis-like lesions in mice models induced by IL-23 is dependent on IL-17 and IL-22 activity [65]. Knockout mice for either IL-17 or IL-22 gene, notwithstanding stimulation with IL-23, does not develop tissue alterations characterizing psoriasiform lesions [65]. We also considered IL- 6 and TNF- $\alpha$ as they might be classified as both psoriasis-related cytokines and adipokines, particularly TNF- $\alpha$, representing a key cytokine in psoriasis pathogenesis and a crucial therapeutic target. We excluded IFN- $\gamma$ as well as IL-12 because not pathogenically relevant. For the same reason we excluded other cytokines (ex., IL-9, IL-19, IL-20, IL-21, IL-24) whose role seems marginal. Regarding adipokines, we focused the attention on those mediators with pro-inflammatory or anti-inflammatory activity that had been associated with psoriasis. However, table 1 also included mediators (both adipokines and cytokines) that are not extensively discussed throughout the text.

\subsection{Role of psoriasis-signature cytokines in increased adiposity}

\section{$2.1 \mathrm{IL}-17$}

A pathogenic link correlating IL-17, psoriasis, and increased adiposity has been proposed, though IL-17 effects on adipose tissue metabolism and gene expression are poorly investigated.

IL-17 is reported to have a protective role against obesity as it inhibits adipogenesis, reduces lipid and glucose uptake acting on both pre-adipocytes and adipocytes [40,41]. Particularly, IL-17 interferes with pre-adipocyte differentiation, downregulating the expression of $\mathrm{C} /$ enhancer binding protein 
(EBP)- $\alpha$ and peroxisome proliferator-activated receptor gamma(PPAR)- $\gamma$, and inhibiting lipid uptake, but not affecting pre-adipocyte proliferation [40]. Along these lines, the absence of IL-17 led to the upregulation of proadipogenic transcription factors (CEBP- $\alpha$ and PPAR- $\gamma$ ), adipocyte-related cytokines (i.e., adiponectin), and genes involved in lipid and glucose metabolism [40]. Of note, in lean IL-17 knock-out (KO) mice, adiponectin serum levels resulted increased, whereas adiponectin suppression was induced by IL-17 in vivo and in vitro [40]. Because adiponectin is exclusively detected in mature adipocytes, the IL-17induced suppression highlights its anti-adipogenic activity. Notwithstanding these data, a positive correlation between IL-17 expression levels and increased adiposity was found, mirroring diet-induced obese mice showing an increased number of circulating IL-17-secreting CD4+ cells. Furthermore, obesity positively correlated with both IL-17 expression and disease severity in IL-17-driven inflammatory mouse models [3]. This positive correlation between obesity and IL-17 expression may influence psoriasis inflammation as suggested by a psoriasis model, wherein increased adiposity aggravates imiquimod-induced psoriasiform dermatitis through the increased expression of both IL-17 and IL22 (five-fold higher than imiquimod-treated lean controls) [53]. Thus, the worsening of imiquimod-induced psoriasiform dermatitis in geneticallydetermined obese mice as well as in high-fat-fed obese mice, reflects the higher prevalence and severity of psoriasis in obese patients, and the impact on therapeutic response and disease severity determined by weight variation [53]. Overall, IL-17A is not proved to profoundly impact on obesity and metabolic status, as suggested by the no-protection from metabolic syndrome and noimprovement of adipose tissue metabolism in established obesity occurring in 
IL-17KO mice [40]. Nevertheless, IL-17 maintains its pro-inflammatory effects inducing adipocyte production of inflammatory mediators including IL-6, whose expression in adipocytes is regulated by an IL-17 signature transcription factor, C/EBP- $\beta$.

\section{$2.2 I L-22$}

Although adipose tissue is known to express the membrane receptor components, namely IL-22R1 and IL-10R2, which transduce IL-22 signals, the impact of IL-22 on metabolism does not seem relevant [41]. Transgenic mice over-expressing IL-22 did not show any significant difference compared to wildtype mice in developing high fat diet-induced increased adiposity [41]. However, a significantly increased number of Th22 cells was detected in the peripheral blood of patients affected by obesity or diabetes, compared to healthy controls [21]. This could partially be explained by the increased serum level of TNF- $\alpha$ and IL-6, which are known as the major drivers of Th22 polarization [45]. Nevertheless, in subjects with increased adiposity compared to lean controls, a higher number of $1 \mathrm{~L}-17$ - and IL-22-producing CD4+ T cells infiltrating adipose tissue was reported [45]. This marked $\mathrm{T}$ cell infiltration was associated with increased plasma concentrations of IL-22 and IL-6 [45]. Relevantly, human adipose tissue CD4+ T cells co-localized with accumulating macrophages, which secrete IL-1 $\beta$ as well as other inflammatory products such as TNF- $\alpha$ [66]. Because IL-1 $\beta$ release is stimulated by IL-22, and IL-1 $\beta$ represents a key inducer of Th17 differentiation, a feed-forward circuit between IL-1 $\beta$-producing macrophages and Th17 cells might be established [66].

\subsection{TNF- $\alpha$}


Physiologically, TNF- $\alpha$ constitutes a restraining cytokine involved in the complex mechanisms of adipose tissue metabolism. Accumulating macrophages in adipose tissue are considered the most relevant source of TNF- $\alpha$, and other proinflammatory molecules and their infiltration positively correlated with increased adiposity [67]. TNF- $\alpha$ stimulates adipocyte leptin synthesis [68], induces lipolysis and inhibits both lipogenesis and anabolic insulin-like growth factor 1 (IGF-1) production [43,69]. Thus, TNF- $\alpha$ could presumably have a protective role against obesity, with mechanisms limiting body mass increase. This evidence may, at least partially, give reason of (i) the enhanced TNF- $\alpha$ levels detected in obese subjects, with putative protective function; (ii) the body weight increase observed in psoriatic patients undergoing treatment with TNF- $\alpha$ inhibitors or other antipsoriatic agents decreasing TNF- $\alpha$ expression levels [56, 70-72]. However, data on TNF- $\alpha$ role in increased adiposity are controversial as it may also contribute to obesity counteracting insulin receptor activity and inhibiting glucose transporter (GLUT)-4, with a consequent enhancement of insulin levels that stimulates the hunger center [68]. Notwithstanding mounting evidence suggesting the TNF- $\alpha$ contribution to insulin resistance, anti-TNF- $\alpha$ binding protein administration failed to improve insulin resistance in diabetic or insulin-resistant patients [73]. Likewise, anti-TNF- $\alpha$ therapies in non-diabetic psoriatic patients did not significantly vary serum concentrations of both leptin and resistin [74]. Overall, the ability of TNF- $\alpha$ blockade in dampening both resistin and leptin levels is still debated and controversial, albeit the antiinflammatory effect of anti-TNF agents is more likely associated with an increased body weight, positively correlating with leptin serum levels, and a presumable reduction of insulin resistance. 


\section{$2.4 I L-6$}

This adipo-cytokine is produced by a plethora of both immune and tissue cells that includes macrophages, dendritic cells, T cells, B cells, keratinocytes, fibroblasts, endothelial cells, and adipocytes. Particularly, it is increasingly expressed in inflammatory conditions such as psoriasis, in metabolic conditions such as obesity-associated insulin resistance $[75,76]$. In obesity setting, $1 \mathrm{~L}-6$ expression mainly derives from adipose tissue, particularly from adipocytes and adipose tissue-derived macrophages, contributing 33\% and 20\%, respectively [67]. In a recent study, adipose tissue-dendritic cells were also demonstrated to express higher levels of IL-6, as well as IL-23 and TGF- $\beta$ [77].

IL-6 has pleiotropic effects acting on a variety of cells. With regard to obesity, lipolysis process, and insulin resistance, its role is still debated. Nevertheless it is reported to (i) suppress lipoprotein lipase activity; (ii) to stimulate energy expenditure; (iii) to show anorexic effects suppressing appetite [78]. Because of its neuroendocrine activity it affects the central nervous system (i) regulating the hypothalamus-pituitary-adrenal axis, thus modulating cortisol release; (ii) stimulating liver secretion of acute-phase proteins; (iii) affecting steroid hormone conversion, altering sex hormone balance and therefore adipose tissue distribution [78, 79].

In a mice model, IL-6 stimulation was demonstrated to interfere with insulin signaling in hepatocytes, favoring insulin resistance [80]. In contrast, IL-6 deficiency was associated with obesity and hepatic inflammation, whereas IL-6 administration reverted insulin resistance [81]. The contrasting data on lipid and glucose metabolism suggest that IL-6 effects may be dependent on its tissuespecific activity and on the localization of its increased expression [82]. 
Beside its activity in regulating insulin resistance and obesity, IL-6 is also crucial for linking these metabolic disorders to inflammation. A diet-induced obese model showed an increased number of IL-17-secreting CD4+ T cells, whose development was dependent on IL-6 stimulation [24]. Indeed, IL-6 KO mice on high-fat diet, despite developing obesity, did not show a Th17-skewed differentiation, indicating that obesity-induced Th17 differentiation was IL-6dependent [24]. Notably, adipose tissue contributes to IL-17-producing T cell development, through adipose-tissue dendritic cell-derived products (i.e., IL-23, TGF- $\beta$, and IL-6) that are involved in IL-17-producing $\mathrm{T}$ cell differentiation and expansion [77].

\subsection{Role of adipokines in psoriasis inflammatory pathway}

\subsection{Leptin}

Compared to the multitude of studies on systemic leptin effects, its role in the skin is poorly investigated. Leptin is mainly secreted by adipocytes and directed to target cells, such as epithelial cells, nervous cells and immune cells expressing its receptor LepR $[27,83]$. Indeed, leptin is known to stimulate keratinocyte proliferation in vitro and to act as a mitogen during skin repair in vivo [84]. In accordance, LepR is particularly expressed in proliferating basal layer and in neo-epithelial keratinocytes during skin repair [84]. It have been shown activation of the classical leptin pathway via JAK/STAT cascade in human HaCaT cells during wound repair, and induction of keratinocyte-derived proinflammatory cytokines, such as IL-6, IL-8 and TNF- $\alpha$ [85]. In addition, leptin is able to alter keratinocytes redox state increasing ROS generation as well as the 
ratio of oxidized/reduced glutathione, and AP-1 activity [86]. This could be linked to the effects of leptin in the psoriatic inflammatory cascade, although findings are controversial. Johnston et al. reported that leptin mRNA levels in involved and uninvolved psoriatic skin were similar to healthy controls [87]. However, in following studies leptin resulted highly represented in psoriatic skin, either in the epidermis, or in the dermis (sweat glands and hair follicles included) $[85,88]$. The authors suggested that, although leptin is involved in wound healing, the strong expression in psoriatic skin respect to controls might be due to an enhanced epidermal turnover. Conversely, multiple leptin systemic effects are well recognized: first of all, its pivotal role in body weight regulation by promoting satiety, and energy consumption [89]. Serum leptin variation represents a response to WAT mass increase, and leptin-deficient mice (ob/ob) are severely obese [90]. Leptin is not just a metabolic hormone, since it acts as a mediator with pleiotropic effects [27]. Indeed, as reported in obese mice, leptin increase is also related to several abnormalities regarding: reproduction, hematopoiesis, angiogenesis, bone homeostasis, lipid and glucose metabolism, as well as innate and adaptive immunity [90-92]. Regarding the innate immunity leptin is able to (i) induce the release of pro-inflammatory mediators (eg. IL-1, IL- 6 and TNF- $\alpha$ ) by monocytes/macrophages; (ii) enhance production of reactive oxygen species (ROS) by neutrophils; (iii) augment NK cell cytotoxicity. Moreover, leptin increases macrophage phagocytosis, neutrophil recruitment, NK cell activation, and promotes DC survival [27], participating to the antimicrobial host defense. In the context of adaptive immunity, leptin is involved in $\mathrm{T}$ cell generation, maturation and survival [93]. It mediates Th1 phenotype differentiation by increasing IFN- $\gamma$, TNF- $\alpha$ and IL-2 production, while 
it inhibits rapamycin-induced Treg cells, by increasing activation of mammalian target of rapamycin (mTOR), which is up-regulated in psoriatic skin as well as in others inflammatory skin conditions (allergic contact dermatitis and atopic dermatitis) $[90,94]$. Moreover, leptin promotes CD4+ cell differentiation into Th17 cells and augments IL-17A production [95]. On the other way, IL-17A also up-regulates leptin release by adipocytes, and the anti-leptin antibody treatment partially antagonizes IL-17A dependent adipogenesis inhibition [96]. Nonetheless, in the context of severe inflammation, leptin may also exert immune-suppressive functions, decreasing Th1 cytokine expression and reducing T cell proliferation [27]. This suggests that the effects of leptin on the immune system may be dependent on the specific milieu.

\subsection{Visfatin}

It was originally called pre-B cell colony-enhancing factor (PBEF) for its capability to augment pre-B-cell colony formation. Only in the last decade, visfatin has been identified as an adipokine, produced principally by adipocytes and working as a bridge between adipose tissue and inflammatory mediators [97]. Visfatin up-regulates human monocytes and endothelial cells production of several mediators, either pro-inflammatory (e.i. IL-6, TNF- $\alpha$ and IL-1 $\beta$ ) or antiinflammatory such as IL-10, IL-1 receptor antagonist (IL-1RA) [97-98]. It acts as chemoattractant for monocytes and lymphocytes. It is able to induce the expression of co-stimulatory molecules, CD80, CD40, and ICAM-1, on monocytes in order to increase T cell activation through a p38 and MEK mediated pathway [99]. Moreover, visfatin inhibits neutrophil apoptosis by caspase-3 as well as caspase-8-dependend mechanism [97]. All these data demonstrate that this adipokine has a prevalently pro-inflammatory function, further reported also in 
human keratinocytes. Indeed, it has been demonstrated that visfatin, in combination with TNF- $\alpha$, stimulates keratinocytes to produce several chemokines (CXCL8, CXCL10, and CCL20), through NF-kB and STAT3 pathway [100], and antimicrobial peptides (CAMP, hBD-2, hBD-3, and S100A7) [101]. This has also been observed in murine models of psoriasis (imiquimod-treated skin), where antimicrobial peptides were enhanced by visfatin [101]. Increased visfatin serum levels in psoriatic patients and positive correlation with Psoriasis Area Severity Index (PASI) have been reported [102], reflecting inflammatory state of these patients.

\subsection{Chemerin}

Chemerin is mostly expressed by fat tissue and liver [103], but also by epithelial barriers, including skin [104-106]. It is released as inactive precursor and converted in its active form by proteinases $[107,108]$, in order to work as ligand for $\mathrm{G}$ protein-associated receptor chemokine-like receptor 1 (CMKLR1) [109]. Chemerin presents a biphasic distribution in normal skin, being over-expressed in the epidermis and down-regulated in the dermis $[104,105]$. The real function of chemerin in the skin is not fully clear, but it has been reported to act as antimicrobial protein [104,106]. Apparently, chemerin mainly works as chemoattractant for CMKLR1+ plasmacytoid dendritic cells (pDCs), macrophages and NK cells, promoting their recruitment at site of tissue damage, and amplifying immune response [107]. Indeed, bacteria and acute phase cytokines, as well as oncostatin M and IL-1 $\beta$, up-regulate its production, while IL-17 and IL22 decrease it [110]. On the other hand, chemerin is induced by TNF- $\alpha$ and IL-1 $\beta$, and its serum levels correlate with TNF- $\alpha$, IL-6, and C reactive protein [111], suggesting that it might also act as a mediator of systemic inflammation. Indeed, 
it has been reported high chemerin circulating levels in psoriasis and psoriatic arthritis patients, with decrease after infliximab therapy [42]. Moreover, uninvolved and involved psoriatic skin contains higher levels of chemerin than normal skin [112]. Accordingly, proteinases required for its activation are overexpressed in involved psoriatic skin [112]. Chemerin is certainly considered as a pro-inflammatory adipokine, but its role in skin diseases needs deeper investigation.

\subsection{Adiponectin}

Adiponectin is highly expressed by mature adipocytes, but also released by skeletal muscle cells, cardiac myocytes, and endothelial cells [30]. Adiponectin, with its multiple oligomeric forms, trimer (LMW), hexamer (MMW), and the high-molecular weight (HMW) [30], (i) regulates energy homeostasis, (ii) reduces insulin resistance and (iii) shows anti-atherogenic, anti-angiogenic, and anti-inflammatory functions [113].

The effect of adiponectin on keratinocytes is yet not fully understood. It has been reported that it inhibits both proliferation and differentiation of keratinocytes. It also suppresses involucrin, TGF $\beta-2$ and -3 expression, and decreases IL-6, IL-8, IL-17, IL-22, and TNF- $\alpha$ secretion by human keratinocytes. In psoriatic patients adiponectin circulating levels are decreased, compared to controls; moreover, they negatively correlated with PASI and the expression of pro-inflammatory mediators, such as IL-6 and TNF- $\alpha[114,115]$. The expression of its receptor ADIPOR1 in psoriatic epidermis is also decreased respect to non lesional or healthy skin [116]. Adiponectin influences skin homeostasis also exerting indirect anti-inflammatory effects: (i) inhibiting TNF- $\alpha$ induced adhesion molecule expression; (ii) interfering with macrophage functions; (iii) inducing 
IL-10 and IL-1RA by human monocytes, macrophages as well as DCs, and (iv) suppressing the production of IFN- $\gamma$ by lipopolysaccharide (LPS)-stimulated human macrophages [113]. In addition, it reduces T-cell proliferation, macrophage phagocytic capability and macrophage TNF- $\alpha$ production [113]. Moreover, adiponectin acts as negative regulator on NK cells, suppressing their IL-2-augmented cytotoxic activity [117]. However, several studies have demonstrated that adiponectin also has pro-inflammatory effects, increasing the production of pro-inflammatory factors such as MMP-3, MMP-9, CCL-2, IL-8, and IL-6 [118-120]. It has been hyphotized that this dual function of adiponectin is isoform-specific: indeed, HWM adiponectin increases IL-6 production in human monocytes, whereas MMW isoform decreases LPS-mediated IL-6 secretion and also induces IL-10 release [121]. Moreoyer, Jung et al. suggested that adiponectin immunoregulatory ability works activating DCs to release pro-inflammatory cytokines that polarize naïve CD4+ T cells into Th1 and Th17 phenotypes [122]. Adiponectin enhances IL-17 expression more than IFN- $\gamma$, indicating that predominantly induce a Th17 response [123]. The complexity of this adipokine and its involyement in inflammatory skin diseases need to be elucidated.

\subsection{Conclusion}

The relationship between increased adiposity and psoriasis is an interesting as well as a challenging topic. The low but chronic grade of inflammation associated to increased adiposity might be involved in the development or in the amplification of chronic dermatoses, either through a direct interaction between adipocytes and keratinocytes or through the indirect modulation of the adaptive immune system. Adipokines are considered specific biomarkers of inflammation 
related to increased adiposity, and they might work as a bridge between fat and skin in psoriasis patients (Figure 1) [124]. Indeed, in these patients, an excess of adipose tissue augments cardiovascular risk and might compromise the therapeutic response $[123,125]$. On the other hand, psoriasis-signature proinflammatory cytokines may alter lipid metabolism, enhancing the risk of increased adiposity and cardiovascular diseases. However, as emerged from the contradictor data analyzed, the mechanism, the temporal link or the biological value of this "pro-inflammatory" connection between inflamed skin and increased adiposity require indeed further investigations.

\subsection{Expert commentary}

Obesity has become a worldwide epidemic health problem, that has taken significant attention to research aimed at understanding the biology of adipocytes and the events occurring in adipose tissue. Several studies show that increased adiposity causes chronic low-grade inflammation that promotes systemic metabolic dysfunction and obesity-linked disorders, such as insulin resistance, type 2 diabetes, atherosclerosis and ischemic heart disease. All these conditions not only reduce life expectancy, but also have enormous economic and societal impact. Increasing epidemiological findings suggests that patients with psoriasis have higher prevalence and incidence of obesity respect to the general population. Better understanding the relationship between psoriasis and increased adiposity is also important for delineating risk factors for all obesitylinked disorders in these patients. It will therefore help to improve future clinical studies that will include adequate adjustments for the presence of obesity as comorbidity among psoriatic patients. Recommendations to reduce 
weight in obese patients with psoriasis may have favorable effects on obesityassociated disorders as well as on psoriasis severity. Moreover, new therapeutic strategies that balance pro- and anti-inflammatory adipokines levels in obese psoriatic patients could have a beneficial role in preventing and/or treating obesity-related metabolic and cardiovascular comorbidities.

\subsection{Five-year view}

Future studies are necessary to better clarify the mechanisms underlying the relation between increased adiposity and psoriasis. Keratinocytes as well as adipocytes are endocrine organ secreting multiple mediators: cytokines and adipokines, respectively. These represent key actors of the chronic inflammatory process involved both in psoriasis and obesity. The role of TNF- $\alpha$ is well known in psoriasis as well as in obesity pathogenesis and several studies have reported the beneficial effects of the anti-TNF- $\alpha$ treatment on psoriasis comorbidity, such as metabolic syndrome. On the contrary, while the role of IL-17 in psoriasis has been elucidated, controversial are the studies reported in the literature on its role in increased adiposity and adipose tissue homeostasis. Then, we suppose that this point will be more investigated in the next studies, focusing mainly on the impact on psoriasis comorbidity of new biologic anti-IL-17 therapies.

\subsection{Key issues}

- Psoriasis is chronic skin disorder associated with several comorbid conditions, including metabolic syndrome.

- The crosstalk between adipose tissue-derived products and psoriasissignature cytokines may contribute to the pathogenesis of both psoriasis and metabolic syndrome. 
- Though cytokine effects on adipose tissue are not fully elucidated and controversial, IL-17 as well as other cytokines alters the homeostasis of adipose tissue.

- Adipokines, particularly chemerin and leptin, showing pro-inflammatory effects, may contribute to psoriasis pathogenesis.

\section{Declaration of Interests}

The authors have no relevant affiliations or financial involvement with any organization or entity with a financial interest in or financial conflict with the subject matter or materials discussed in the manuscript. This includes employment, consultancies, honoraria, stock ownership or options, expert testimony, grants or patents received or pending, or royalties.

\section{References}

Reference annotations

* Of interest

** Of considerable interest

1. Scha"fer T. Epidemiology of psoriasis. Review and the German perspective.

Dermatology 2006;212:327-37

2. Lowes MA, Suárez-Fariñas M, Krueger JG. Immunology of psoriasis. Annu Rev Immunol 2014;32:227-55

3. Davidovici BB, Sattar N, Prinz J, et al. Psoriasis and systemic inflammatory diseases: potential mechanistic links between skin disease and co-morbid conditions. J Invest Dermatol 2010;130:1785-96

4. Balato N, Balato A, Gallo L, et al. Psoriasis and osteoporosis: data from a Southern Italian population. Arch Osteoporos 2012;7:321-3 
5. Balato N, Napolitano M, Ayala F, et al. Nonalcoholic fatty liver disease, spleen and psoriasis: New aspects of low-grade chronic inflammation. World J Gastroenterol 2015 14;21:6892-7

6. Sales R, Torres T. Psoriasis and metabolic syndrome. Acta Dermatovenerol Croat 2014;22(3):169-74

7. Neimann AL, Shin DB, Wang X, et al. Prevalence of cardiovascular risk factors in patients with psoriasis. J Am Acad Dermatol 2006;55:829-35

8. Mebazaa A, El Asmi M, Zidi W, et al. Metabolic syndrome in Tunisian psoriatic patients: prevalence and determinants. J Eur Acad Dermatol Venereol 2011;25:705-9

9. Gisondi P, Tessari G, Conti A, et al. Prevalence of metabolic syndrome in patients with psoriasis: a hospital-based case-control study. $\mathrm{Br}$ J Dermatol $2007 ; 157: 68-73$

10. Sommer DM, Jenisch S, Suchan M, et al. Increased prevalence of the metabolic syndrome in patients with moderate to severe psoriasis. Arch Dermatol Res 2006;298:321-8

11. Cohen AD, Gilutz $H$, Henkin $Y$, et al. Psoriasis and the metabolic syndrome. Acta Derm Venereol 2007;87:506-9

12. Zindanci I, Albayrak O, Kavala M, et al. Prevalence of metabolic syndrome in patients with psoriasis. Scientific World Journal 2012;2012:312-463

13. Love TJ, Qureshi AA, Karlson EW, et al. Prevalence of the metabolic syndrome in psoriasis: results from the National Health and Nutrition Examination Survey, 2003-2006. Arch Dermatol 2011;147:419-24

14. Quaranta M, Burden AD, Griffiths CE, et al. Differential contribution of CDKAL1 variants to psoriasis, Crohn's disease and type II diabetes. Genes Immun 
2009;10:654-8

15. Wolf N, Quaranta M, Prescott NJ, et al . Psoriasis is associated with pleiotropic susceptibility loci identified in type II diabetes and Crohn disease. J Med Genet 2008;45:114-6

16. Barrea L, Macchia PE, Tarantino G, et al. Nutrition: a key environmental dietary factor in clinical severity and cardio-metabolic risk in psoriatic male patients evaluated by 7-day food-frequency questionnaire. J Transl Med 2015 $16 ; 13: 303$

17. Barrea L, Balato N, Di Somma C, et al. Nutrition and psoriasis: is there any association between the severity of the disease and adherence to the Mediterranean diet? J Transl Med 2015 27;13:18

18. Guida B, Napoleone A, Trio R, et al, Energy-restricted, n-3 polyunsaturated fatty acids-rich diet improves the clinical response to immuno-modulating drugs in obese patients with plaque-type psoriasis: a randomized control clinical trial. Clin Nutr 2014;33:399-405

19. Balato A, Megna M. Adipose tissue and cutaneous inflammation. Clinical and basic Immunodermatology. Gaspari A, Tyring S, Kaplan D. (Eds.). Elsivier (in press).

20. Ouchi N, Parker JL, Lugus JJ, Walsh K. Adipokines in inflammation and metabolic disease. Nat Rev Immunol 2011;11:85-97

21. Zhao R, Tang D, Yi S, et al. Elevated peripheral frequencies of Th22 cells: a novel potent participant in obesity and type 2 diabetes. PLoS One 2014;9:e85770 22. Osborn O, Olefsky JM. The cellular and signaling networks linking the immune system and metabolism in disease. Nat Med 2012;18:363-74 
23. Balato A, Unutmaz D, Gaspari AA. Natural killer T cells: an unconventional Tcell subset with diverse effector and regulatory functions. J Invest Dermatol 2009;129:1628-42

24. Winer S, Paltser G, Chan Y, Tsui H, et al. Obesity predisposes to Th17 bias. Eur J Immunol 2009;39:2629-35

\section{** Obesity-related predisposition to Th17-driven inflammation}

25. Hutcheson J. Adipokines influence the inflammatory balance in autoimmunity. Cytokine. 2015;75:272-9.

26. Lago F, Dieguez C, Gomez-Reino J, Gualillo O. Adipokines as emerging mediators of immune response and inflammation. Nat Clin Pract Rheumatol $2007 ; 3: 716-24$

27. Fernández-Riejos P, Najib S, Santos-Alvarez J, et al. Role of leptin in the activation of immune cells. Mediators Inflamm 2010;2010:568343

28. Ferrante AW Jr. Obesity-induced inflammation: a metabolic dialogue in the language of inflammation. J Intern Med 2007;262:408-14

29. Galic S, Oakhill JS, Steinberg GR. Adipose tissue as an endocrine organ. Mol Cell Endocrinol 2010;316:129-39

30. Richards AA, Stephens T, Charlton HK, et al. Adiponectin multimerization is dependent on conserved lysines in the collagenous domain: evidence for regulation of multimerization by alterations in posttranslational modifications. Mol Endocrinol 2006;20:1673-87

31. Jamaluddin MS, Weakley SM, Yao Q, Chen C. Resistin: functional roles and therapeutic considerations for cardiovascular disease. $\mathrm{Br} \mathrm{J}$ Pharmacol $2012 ; 165: 622-32$ 
32. Schelbert KB. Comorbidities of obesity. Prim Care 2009;36:271-85

33. Bertrand C, Valet P, Castan-Laurell I. Apelin and energy metabolism. Front Physiol 2015;6:115

34. Schultz S, Beck-Sickinger AG. Chemerin and vaspin: possible targets to treat obesity? ChemMedChem. 2013;8:549-59

35. Zabetian-Targhi F, Mahmoudi MJ, Rezaei N, Mahmoudi M. Retinol binding protein 4 in relation to diet, inflammation, immunity, and cardiovascular diseases. Adv Nutr 2015;6:748-62

36. Cinti S, Mitchell G, Barbatelli G, et al. Adipocyte death defines macrophage localization and function in adipose tissue of obese mice and humans. J Lipid Res $2005 ; 46: 2347-55$

37. Cypess AM, Lehman S, Williams G, et al. Identification and importance of brown adipose tissue in adult humans. N Engl Med 2009;360:1509-17

38. Ford ES, Mokdad AH. Epidemiology of obesity in the western hemisphere. J Clin Endocrinol Metab 2008;93:S1-8

39. Antuna-Puente B, Feve B, Fellahi S, Bastard JP. Adipokines: the missing link between insulin resistance and obesity. Diabetes Metab 2008;34:2-11

40. Zúñiga LA, Shen WJ, Joyce-Shaikh B, et al. IL-17 regulates adipogenesis, glucose homeostasis, and obesity. J Immunol 2010;185(11):6947-59

41. Wang Z, Yang L, Jiang Y, et al. High fat diet induces formation of spontaneous liposarcoma in mouse adipose tissue with overexpression of interleukin 22. PLoS One 2011;6:e23737

42. Gisondi P, Lora V, Bonauguri C, et al. Serum chemerin is increased in patients with chronic plaque psoriasis and normalizes following treatment with infliximab. Br J Dermatol 2013;168: 749-755 
43. Jin D, Sun J, Huang J, He Y, Yu A, Yu X, Yang Z. TNF- $\alpha$ reduces g0s2 expression and stimulates lipolysis through PPAR- $\gamma$ inhibition in 3T3-L1 adipocytes. Cytokine 2014;69:196-205

44. Saraceno R, Schipani C, Mazzotta A, et al. Effect of anti-tumor necrosis factoralpha therapies on body mass index in patients with psoriasis. Pharmacol Res $2008 ; 57: 290-5$

45. Bertola A, Ciucci T, Rousseau D, et al. Identification of adipose tissue dendritic cells correlated with obesity-associated insulin-resistance and inducing Th17 responses in mice and patients. Diabetes 2012;61:2238-47

46. Sterry W, Strober BE, Menter A; International Psoriasis Council. Obesity in psoriasis: the metabolic, clinical and therapeutic implications. Report of an interdisciplinary conference and review. Br J Dermatol 2007;157:649-55

* Overview about evidence supporting the association between obesity and psoriasis

47. Kintscher U, Hartge $M$, Hess $K$, et al. T-lymphocyte infiltration in visceral adipose tissue: a primary event in adipose tissue inflammation and the development of obesity-mediated insulin resistance. Arterioscler Thromb Vasc Biol 2008;28:1304-10

48. Duffaut C, Galitzky J, Lafontan M, Bouloumié A. Unexpected trafficking of immune cells within the adipose tissue during the onset of obesity. Biochem

Biophys Res Commun 2009;384:482-5

** Contribution of immune cells to obesity 
49. Fabbrini E, Cella M, McCartney SA, et al. Association between specific adipose tissue CD4+ T-cell populations and insulin resistance in obese individuals. Gastroenterology 2013;145:366-74

50. Cipolletta D, Kolodin D, Benoist C, Mathis D. Tissular T(regs): a unique population of adipose-tissue-resident Foxp3+CD4+ $T$ cells that impacts organismal metabolism. Semin Immunol 2011;23:431-437

51. Paller AS, Mercy K, Kwasny MJ, et al. Association of pediatric psoriasis severity with excess and central adiposity: an international cross-sectional study. JAMA Dermatol 2013;149:166-76

* Clinical evidence supporting the association between psoriasis and central obesity in pediatric population

52. Matteo Megna and Maddalena Napolitano, Balato A, Scalvenzi M, et al. Psoriasis in Children: A Review Curr Pediatr Rev 2015;11:10-26

53. Kanemaru K, Matsuyuki A, Nakamura Y, Fukami K. Obesity exacerbates imiquimod-induced psoriasis-like epidermal hyperplasia and interleukin-17 and interleukin-22 production in mice. Exp Dermatol 2015;24:436-42

**Key-evidence in mice model demonstrating that worsening of psoriasislike inflammation may be caused by obesity

54. Gisondi P, Del Giglio M, Di Francesco V, et al. Weight loss improves the response of obese patients with moderate-to-severe chronic plaque psoriasis to low-dose cyclosporine therapy: a randomized, controlled, investigator-blinded clinical trial. Am J Clin Nutr 2008;88: 1242-7

*Correlation between BMI variation and response to antipsoriatic treatment 
55. Di Minno MN, Peluso R, Iervolino S, et al. Obesity and the prediction of minimal disease activity. A prospective study in psoriatic arthritis. Arthritis Care Res (Hoboken) 2013;65:141-7

56. Bardazzi F, Balestri R, Baldi E, et al. Correlation between BMI and PASI in patients affected by moderate to severe psoriasis undergoing biological therapy. Dermatol Ther 2010;23:14-9

57. Takahashi H, Tsuji H, Takahashi I, et al. Plasma adiponectin and leptin levels in Japanese patients with psoriasis. Br J Dermatol. 2008;159(5):1207-8.

58. Baran A, Flisiak I, Jaroszewicz J, Świderska M. Effect of psoriasis activity on serum adiponectin and leptin levels. Postep Derm Alergol 2015; 32 (2): 101-106 59. Huang H, Shen E, Tang S, et al. Increased serum resistin levels correlate with psoriasis: a meta-analysis. Huang et al. Lipids in Health and Disease (2015) 14:44 60. Takahashi H, Tsuji H, Ishida-Yamamoto A, Iizuka H. Serum level of adiponectin increases and those of leptin and resistin decrease following the treatment of psoriasis. J Dermatol. 2013; 40(6):475-6.

61. Coban M, Tasli L, Turgut S, et al. Association of Adipokines, Insulin Resistance, Hypertension and Dyslipidemia in Patients with Psoriasis Vulgaris. Ann Dermatol 2016; 28(1): 74-79

62. Mariani F, Roncucci L. Chemerin/chemR23 axis in inflammation onset and resolution. Inflamm Res 2015;64:85-95.

63. Lowes MA, Russell CB, Martin DA, Towne JE, Krueger JG. The IL-23/T17 pathogenic axis in psoriasis is amplified by keratinocyte responses. Trends Immunol. 2013;34:174-81.

64. Chiricozzi A. Pathogenic role of IL-17 in psoriasis and psoriatic arthritis. Actas Dermosifiliogr. 2014; 105: 9-20. 
65. Rizzo HL, Kagami S, Phillips KG, Kurtz SE, Jacques SL, Blauvelt A. IL-23mediated psoriasis-like epidermal hyperplasia is dependent on IL-17A. J Immunol. 2011;186:1495-502.

66. Dalmas E, Venteclef N, Caer C, et al. T cell-derived IL-22 amplifies IL-1 $\beta$ driven inflammation in human adipose tissue: relevance to obesity and type 2 diabetes. Diabetes 2014;63:1966-77

67. Chawla A, Nguyen KD, Goh Y.P S. Macrophage-mediated inflammation in metabolic disease. Nat Rev Immunol 2011;11:738-49

68. Gustafson B. Adipose tissue, inflammation and atherosclerosis. J Atheroscler Thromb 2010;17:332-41

69. Grohmann M, Sabin M, Holly J, Shield J, Crowne E, Stewart C Characterization of differentiated subcutaneous and visceral adipose tissue from children: the influences of TNF-alpha and IGF-I. LLipid Res 2005;46:93-103.

70. Owczarczyk-Saczonek A, Placek W, Rybak-d'Obyrn J, Wygonowska E. Influence of ustekinumab on body weight of patients with psoriasis: an initial report. Postepy DermatolAlergol 2014;31:29-31

71. Tan E, Baker C, Foley P. Weight gain and tumour necrosis factor-alpha inhibitors in patients with psoriasis. Australas J Dermatol 2013;54:259-63

72. Renzo LD, Saraceno R, Schipani C, et al. Prospective assessment of body weight and body composition changes in patients with psoriasis receiving antiTNF- $\alpha$ treatment. Dermatol Ther 2011;24:446-51

73. Peluso I, Palmery M. The relationship between body weight and inflammation: Lesson from anti-TNF- $\alpha$ antibody therapy. Hum Immunol $2016 ; 77: 47-53$ 
74. Pina T, Genre F, Lopez-Mejias R, et al. Relationship of leptin with adiposity and inflammation and resistin with disease severity in psoriatic patients undergoing anti-TNF-alpha therapy. J Eur Acad Dermatol Venereol 2015;29:1995-2001

75. Vasilopoulos Y, Sourli F, Zafiriou E, et al. High serum levels of HIF-1 $\alpha$ in psoriatic patients correlate with an over-expression of IL-6. Cytokine 2013; $62: 38-9$

76. Tangvarasittichai S, Pongthaisong S, Tangvarasittichai O. Tumor Necrosis Factor-A, Interleukin-6, C-Reactive Protein Levels and Insulin Resistance Associated with Type 2 Diabetes in Abdominal Obesity Women. Indian J Clin Biochem 2016;31:68-74

77. Chen $Y$, Tian J, Tian $X$, et al. Adipose tissue dendritic cells enhances inflammation by prompting the generation of Th17 cells. PLoS One 2014;9: e92450

78. Wernstedt I, Edgley A, Berndtsson A, et al. Reduced stress- and cold-induced increase in energy expenditure in interleukin-6-deficient mice. Am J Physiol Regul Integr Comp Physiol 2006;291:R551-7

79. Küçük M, Altınkaya SÖ, Nergiz S, et al. Interleukin-6 levels in relation with hormonal and metabolic profile in patients with polycystic ovary syndrome. Gynecol Endocrinol 2014;30:423-7

80. Dou L, Zhao T, Wang L, et al. miR-200s contribute to interleukin-6 (IL-6)induced insulin resistance in hepatocytes. J Biol Chem 2013;288:22596-606

81. Matthews VB, Allen TL, Risis S, et al. Interleukin-6-deficient mice develop hepatic inflammation and systemic insulin resistance. Diabetologia 2010;53: 2431-41 
82. Kwon H, Pessin JE. Adipokines mediate inflammation and insulin resistance. Front Endocrinol (Lausanne) 2013;4:71

83. Stern JH, Rutkowski JM, Scherer PE. Adiponectin, Leptin, and Fatty Acids in the Maintenance of Metabolic Homeostasis through Adipose Tissue Crosstalk. Cell Metab 2016;23:770-84

84. Tadokoro S, Ide S, Tokuyama R, et al. Leptin promotes wound healing in the skin. PLoS One 2015;10:e0121242

85. Xue K, Liu H, Jian Q, et al. Leptin induces secretion of pro-inflammatory cytokines by human keratinocytes in vitro-a possible reason for increased severity of psoriasis in patients with a high body mass index. Exp Dermatol 2013;22:406-10

86. Savini I, Catani MV, Rossi A, et al. Vitamin C recycling is enhanced in the adaptive response to leptin-induced oxidative stress in keratinocytes. Endocrinology 2008;149:5189-98

87. Johnston A, Arnadottir S, Gudjonsson JE, et al. Obesity in psoriasis: leptin and resistin as mediators of cutaneous inflammation. Br J Dermatol 2008;159:342-50 88. Cerman AA, Bozkurt S, Sav A, et al. Serum leptin levels, skin leptin and leptin receptor expression in psoriasis. Br J Dermatol 2008;159:820-6

89. Moehlecke M, Canani LH, Silva LO, et al. Determinants of body weight regulation in humans. Arch Endocrinol Metab 2016;[Epub ahead of print]

90. Versini M, Jeandel PY, Rosenthal E, Shoenfeld Y. Obesity in autoimmune diseases: Not a passive bystander. Autoimmun Rev 2014;13:981-1000

91. Ducy $\mathrm{P}$, Amling M,Takeda S, Prieme M, SchillingAF, Beil FT, et al. Leptin inhibits bone formation through a hypothalamic relay: a central control of bone mass. Cell 2000;100:197-207 
92. Procaccini C, Jirillo E, Matarese G. Leptin as an immunomodulator. Mol Aspects Med 2012;33:35-45

93. Lam QLK, Liu S, Cao X, et al. Involvement of leptin signaling in the survival and maturation of bone marrow-derived dendritic cells. Eur J Immunol 2006; $36: 3118-30$

94. Balato A, Di Caprio R, Lembo S, et al. Mammalian Target of Rapamycin in Inflammatory Skin Conditions. Eur J Inflamm 2014;12:341-50

95. Orlova EG, Shirshev SV. Role of leptin and ghrelin in induction of differentiation of IL-17-producing and T-regulatory cells. Bull Exp Biol Med $2014 ; 156: 819-22$

96. Noh M. Interleukin-17A increases leptin production in human bone marrow mesenchymal stem cells. Biochem Pharmacol 2012;83:661-70

97. Moschen AR, Kaser A, Enrich B, et al, Visfatin, an adipocytokine with proinflammatory and immunomodulating properties. J Immunol 2007;178:1748-58

98. Curat CA, Wegner V, Sengenes C, et al. Macrophages in human visceral adipose tissue: increased accumulation in obesity and a source of resistin and visfatin. Diabetologia 2006;49:744-7

99. Stofkova A. Resistin and visfatin: regulators of insulin sensitivity, inflammation and immunity. Endocr Regul 2010;44:25-36

100. Kanda N, Hau CS, Tada Y, et al. Visfatin enhances CXCL8, CXCL10, and CCL20 production in human keratinocytes. Endocrinology 2011;152:3155-64

101. Hau CS, Kanda N, Noda S, et al. Visfatin enhances the production of cathelicidin antimicrobial peptide, human $\beta$-defensin- 2 , human $\beta$-defensin- 3 , and 
S100A7 in human keratinocytes and their orthologs in murine imiquimodinduced psoriatic skin. Am J Pathol 2013;182:1705-17
102. Okan
G, Baki
AM, Yorulmaz
E, Doğru-Abbasoğlu
S, Vural P. Serum Visfatin, Fetuin-A, and Pentraxin 3 Levels in Patients With Psoriasis and Their Relation to Disease Severity. J Clin Lab Anal 2015;[Epub ahead of print] 103. Zabel BA, Kwitniewski M, Banas $M$, et al. Chemerin regulation and role in host defense. Am J Clin Exp Immunol 2014;3:1-19

104. Albanesi C, Scarponi C, Pallotta S, et al. Chemerin expression marks early psoriatic skin lesions and correlates with plasmacytoid dendritic cell recruitment. J Exp Med 2009;206:249-58

105. Banas M, Zabieglo K, Kasetty G, et al. Chemerin is an antimicrobial agent in human epidermis. PLoS One 2013;8:e58709

106. Luangsay S, Wittamer V, Bondue B, et al. Mouse ChemR23 is expressed in dendritic cell subsets and macrophages, and mediates an anti-inflammatory activity of chemerin in alung disease model. J Immunol 2009;183:6489-99

107. Kulig P, Kantyka T, Zabel BA, et al. Regulation of chemerin chemoattractant and antibacterial activity by human cysteine cathepsins. J Immunol 2011;187:1403-10

108. Kulig P, Zabel BA, Dubin G, et al. Staphylococcus aureus-derived staphopain B, a potent cysteine protease activator of plasma chemerin. J Immunol 2007; 178:3713-20

109. Davenport AP, Alexander SP, Sharman JL, et al. International Union of Basic and Clinical Pharmacology. LXXXVIII. G protein-coupled receptor list: recommendations for new pairings with cognate ligands. Pharmacol Rev $2013 ; 65: 967-86$ 
110. Banas M, Zegar A, Kwitniewski M, et al. The expression and regulation of chemerin in the epidermis. PLoS One 2015;10:e0117830

111. Parlee SD, Ernst MC, Muruganandan S, et al Serum chemerin levels vary with time of day and are modified by obesity and tumor necrosis factor-\{alpha\}. Endocrinology 2010;151:2590-2

112. Skrzeczyńska-Moncznik J, Wawro K, Stefańska A, Oleszycka E, et al. Potential role of chemerin in recruitment of plasmacytoid dendritic cells to diseased skin. Biochem Biophys Res Commun 2009;380:323-7

113. Wang ZV, Scherer PE. Adiponectin, the past two decades. J Mol Cell Biol 2016;[Epub ahead of print]

114. Kaur S, Zilmer K, Kairane C, Kals M, Zilmer M. Clear differences in adiponectin level and glutathione redox status revealed in obese and normalweight patients with psoriasis. Br JDermatol 2008;159:1364-7

115. Shibata S, Saeki H, Tada Y, Karakawa M, Komine M, Tamaki K. Serum high molecular weight adiponectin levels are decreased in psoriasis patients. J Dermatol Sci 2009;55:62-3

116. Takahashi H, Honma M, Ishida-Yamamoto A, Iizuka H. Adiponectin and leptin modulate cell proliferation and cytokine secretion of normal human keratinocytes and T lymphocytes. J Dermatol Sci 2010;59:143-5

117. Kim MS, Pak YK, Jang PG, et al. Role of hypothalamic Foxo1 in the regulation of food intake and energy homeostasis. Nat Neurosci 2006;9:901-6

118. Tong KM, Chen CP, Huang KC, et al. Adiponectin increases MMP-3 expression in human chondrocytes through AdipoR1 signaling pathway. J Cell Biochem 2011;112:1431-40 
119. Lago R, Gomez R, Otero M, et al. A new player in cartilage homeostasis: adiponectin induces nitric oxide synthase type II and pro-inflammatory cytokines in chondrocytes. Osteoarthr Cartilage/OARS, Osteoarthr Res Soc 2008;16:1101-9

120. Gomez R, Scotece M, Conde J, et al. Adiponectin and leptin increase IL-8 production in human chondrocytes. Ann Rheum Dis 2011;70:2052-4

121. Neumeier M, Weigert J, Schäffler A, et al. Different effects of adiponectin isoforms in human monocytic cells. J Leukoc Biol 2006;79:803-8

122. Jung MY, Kim HS, Hong HJ, Youn BS, Kim TS. Adiponectin induces dendritic cell activation via PLC $\gamma / \mathrm{JNK} / \mathrm{NF}-\kappa \mathrm{B}$ pathways, leading to Th1 and Th17 polarization. J Immunol 2012;188:2592-601

123. Molinelli E, Campanati A, Ganzetti G, Offidani A. Biologic Therapy in Immune Mediated Inflammatory Disease: Basic Science and Clinical Concepts. Curr Drug Saf $2016 ; 11: 35-43$

124. Danilenko DM. Review paper: preclinical models of psoriasis. Vet Pathol. $2008 ; 45: 563-75$

125. Campanati A, Ganzetti G, Giuliodori K, et al. Serum levels of adipocytokines in psoriasis patients receiving tumor necrosis factor- $\alpha$ inhibitors: results of a retrospective analysis. Int J Dermatol 2015;54:839-45

Figure 1. Crosstalk between skin and adipose tissue in psoriasis. 


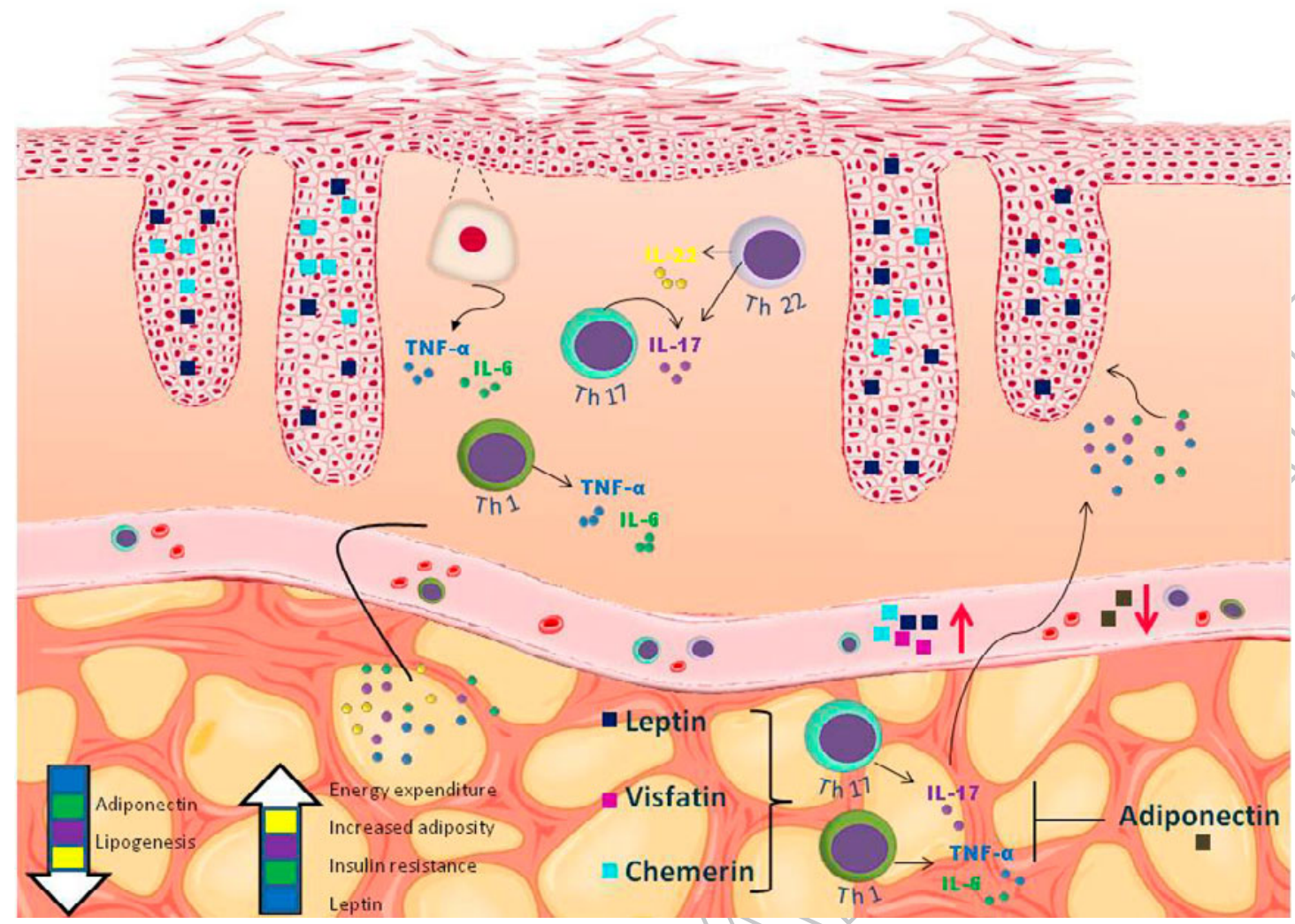

Psoriasis-signature cytokines, such as TNF- $\alpha$, IL-17, IL-22 and IL-6, have effects on adipose tissue being involved in key mechanisms of lipidic metabolism, including increased adiposity and insulin resistance. Secreted adipokines, such as leptin, visfatin and chemerin have pro-inflammatory effects, amplifying the immune response, through Th17 and Th1; whereas adiponectin shows antiinflammatory effects. Moreover, high circulating levels of leptin, visfatin and chemerin have been reported in psoriatic patients, whereas adiponectin is decreased. In the plaque an enhancement of leptin and chemerin protein expression has been found. 
Table I. Main features of relevant adipokines and cytokines.

\begin{tabular}{llc}
\hline Adipokines & \multicolumn{1}{c}{ Main role } & Site of production \\
\hline & \multicolumn{1}{c}{ Adipokines with prevalent hormonal activity } & \\
\hline Leptin & $\begin{array}{l}\text { Stimulates satiety, lipolysis, glucose and lipid } \\
\text { metabolism, enhances insulin sensitivity [27] }\end{array}$ & Adipocyte \\
\hline Visfatin & Enhances insulin sensitivity, induces lipogenesis [28] & Adipocyte \\
\hline Chemerin & Stimulates adipogenesis and insulin resistance [29] & Adipocyte \\
\hline Adiponectin & $\begin{array}{l}\text { Regulates glucose metabolism, enhances insulin } \\
\text { sensitivity [30] }\end{array}$ & Adipocyte \\
\hline Resistin & Induces insulin resistance [31] & Adipocyte, macrophage \\
\hline Adipsin & Inhibits lipolysis [32] & Adipocyte, macrophage \\
\hline Apelin & Inhibits insulin secretion [33] & Adipocyte, macrophage \\
\hline Vaspin & Enhances insulin resistance [34] & Adipocyte \\
\hline Omentin & Modulates insulin activity [32] & Macrophage \\
\hline Lipocalin 2 & Induces insulin sensitivity [32] & Adipocyte, macrophage \\
\hline & & Adipocyte \\
\hline
\end{tabular}

Adipo-cytokines involved in inflammatory response

\begin{tabular}{llc} 
IL-6 & $\begin{array}{l}\text { Pro-inflammatory cytokines; stimulates lipolysis, } \\
\text { reduces insulin and leptin activity [36] }\end{array}$ & Adipocyte, macrophage, \\
\hline TNF- $\boldsymbol{\alpha}$ & $\begin{array}{l}\text { Pro-inflammatory cytokines; stimulates lipolysis, } \\
\text { induces insulin resistance [36] }\end{array}$ & $\begin{array}{l}\text { endothelial stromal cell } \\
\text { Adipocyte, macrophage }\end{array}$ \\
\hline STNF-RII & $\begin{array}{l}\text { Pro-inflammatory cytokines; stimulates lipolysis, } \\
\text { induces insulin resistance [37] }\end{array}$ & Adipocyte, macrophage \\
\hline IL-1RA & $\begin{array}{l}\text { Anti-inflammatory cytokines; reduces insulin } \\
\text { sensitivity [38] }\end{array}$ & Macrophage \\
\hline IL-10 & Anti-inflammatory cytokine [38] & Adipocyte, macrophage \\
\hline IL-8 & Pro-inflammatory chemokine [32] & Endothelial stromal cell \\
\hline
\end{tabular}

MCP-1

Pro-inflammatory chemokine; stimulates lipolysis, induces insulin resistance [33]

Adipocyte, macrophage

Adipokines with vascular activity

PAI-1
Pro-thrombotic activity, enhances insulin resistance [39]
Endothelial stromal cell

Adipokines with lipid metabolism activity 


\section{Adipokine as acute phase protein}

CRP Stimulates cytokine and adhesion molecule expression

Stimulates monocyte chemotaxis and adhesion molecule expression [34]

Adipocyte

SAA

Psoriasis-signature cytokines involved in lipid and glucose metabolism

IL-17

Inhibits adipogenesis, reduces lipid and glucose uptake $[40,41]$

TNF- $\alpha$

Presumably plays a protective role against obesity $[43,44]$

Suppresses lipoprotein lipase activity; stimulates energy expenditure; drive Th17 development [45]

Role in obesity and insulin resistance to be defined

$[40,45]$

CD4+ T cells, CD8+ T cells, innate lymphoid cells 3 , mast cells, neutrophils, $\gamma \delta$ T cells

Adipose tissue cells, dendritic cells, endothelial cells, fibroblasts, keratinocytes, macrophages, mast cells, neutrophils, T cells

Adipose tissue cells, keratinocytes,endothelial cells, fibroblasts, macrophages, dendritic cells, T cells

IL-22
CD + T cells, $\mathrm{CD} 8+\mathrm{T}$ cells, $\gamma \delta$

$\mathrm{T}$ cells, innate lymphoid cells 3 , mast cells 
Table 2. Main effects of psoriasis-signature cytokines on increased adiposity as well as of adipokines on psoriasis pathogenesis.

\begin{tabular}{|c|c|}
\hline Psoriasis-signature cytokines & Increased adiposity \\
\hline IL-17 & $\begin{array}{l}\text { - inhibits adipogenesis }[40,41] \\
\text { - reduces lipid and glucose uptake }[40,41] \\
\text { - suppresses adiponectin production }[40] \\
\text { - correlates positively with increased adiposity }[3,53] \\
\text { - induces adipocyte production of IL-6 }[40]\end{array}$ \\
\hline IL-22 & $\begin{array}{l}\text { - high number of IL-22-producing CD4+ T cells infiltrating } \\
\text { adipose in increased adiposity [45] }\end{array}$ \\
\hline TNF- $\alpha$ & $\begin{array}{l}\text { - induces lipolysis and inhibits lipogenesis [43] } \\
\text { - stimulates adipocyte leptin synthesis [68] } \\
\text { - contributes to insulin resistance [68] }\end{array}$ \\
\hline IL-6 & $\begin{array}{l}\text { - suppress lipoprotein lipase activity [78] } \\
\text { - stimulates energy expenditure [78] } \\
\text { - show anorexic effects suppressing appetite [78] } \\
\text { - favors insulin resistance [80] }\end{array}$ \\
\hline Adipokines & Psoriasis \\
\hline Leptin & $\begin{array}{l}\text { - high circulating levels in psoriasis patients }[88] \\
\text { - mRNA levels in involyed and uninvolved psoriatic skin are } \\
\text { similar to healthy controls }[87] \\
\text { - protein levels are enhanced in psoriatic skin }[85,88]\end{array}$ \\
\hline Visfatin & $\begin{array}{l}\text { - high serum levels in psoriatic patients and positive correlation } \\
\text { with PASI [102] }\end{array}$ \\
\hline Chemerin & $\begin{array}{l}\text { - high circulating levels in psoriasis and psoriatic arthritis } \\
\text { patients, with decrease after infliximab therapy [42] } \\
\text { high protein levels in uninvolved and involved psoriatic skin } \\
\text { [112] }\end{array}$ \\
\hline Adiponectin & $\begin{array}{l}\text { - circulating levels are decreased in psoriatic patients and } \\
\text { negatively correlate with PASI and the expression of IL- } 6 \text { and } \\
\text { TNF- } \alpha[114,115] \\
\text { - the expression of its receptor ADIPOR1 is decreased in psoriatic } \\
\text { epidermis [116] }\end{array}$ \\
\hline
\end{tabular}

PASI, Psoriasis Area Severity Index

IL, interleukin

TNF, tumor necrosis factor

ADIPOR, adiponectin receptor 